\title{
Local anaesthesia for fibreoptic bronchoscopy: transcricoid injection or the "spray as you go" technique?
}

A R Webb, S S D Fernando, H R Dalton, J E Arrowsmith, M A Woodhead, A R C Cummin

\begin{abstract}
Local anaesthesia for fibreoptic bronchoscopy should be given by a safe technique that is not unpleasant to the patient and should provide acceptable conditions for the bronchoscopist. Single injection transcricoid local anaesthesia was compared with the "spray as you go" technique in patients having day case fibreoptic bronchoscopy. Patients were randomised to receive either $100 \mathrm{mg}$ lignocaine by a single cricothyroid puncture or $240 \mathrm{mg}$ lignocaine instilled through the bronchoscope under direct vision. Further doses were given by the operator to both groups as required. The 30 patients receiving transcricoid lignocaine coughed less (3.56 (SD 3.1) coughs/min) than the 32 patients receiving lignocaine through the bronchoscope $(5.89(4.8) / \mathrm{min})$ despite receiving a lower total dose of lignocaine (322 (25.9) v 451 (20.9) mg). Cricothyroid puncture was not associated with any complications and was not unpleasant for the patients.
\end{abstract}

Lignocaine is the most commonly used local anaesthetic agent for fibreoptic bronchoscopy and has a wide margin of safety. It has been suggested that the total dose should be limited to $300-400 \mathrm{mg}^{12}$ as absorption of lignocaine from the respiratory mucosa is known to be rapid. ${ }^{3}$ Although no direct correlation can be made between plasma concentrations and the dose of lignocaine used for topical anaesthesia of the mucous membranes, ${ }^{45}$ higher plasma concentrations may be associated with adverse effects, such as muscular irritability, convulsions, or coma.

There are several techniques for anaesthetising the vocal cords and tracheobronchial tree, each with its own potential advantages and disadvantages. Day case diagnostic fibreoptic bronchoscopies are performed at $\mathrm{St}$ George's Hospital under light sedation and local anaesthesia. ${ }^{6}$ Topical lignocaine has been applied by the "spray as you go" technique with direct instillation of $2-4 \%$ solutions on to the vocal cords and tracheobronchial mucosa. The nasal mucosa is anaesthetised with lignocaine gel. ${ }^{7}$ The dose of lignocaine applied to the tracheobronchial mucosa under direct vision is $240 \mathrm{mg}$, though there may be wastage because some of the solution applied to the vocal cords may be swallowed. Further doses of lignocaine are used as necessary if coughing occurs.

Other workers use the transcricoid route for local anaesthesia of the vocal cords and tracheobronchial mucosa and have found it to be safe and well tolerated. ${ }^{89}$ Lower doses of local anaesthetic are generally used with this technique but there have been few direct comparisons of the transcricoid route with the "spray as you go" technique.

The better local anaesthetic technique would require a lower dose of local anaesthetic. It would be safe and not unpleasant for the patient and would provide acceptable conditions for the bronchoscopist. ${ }^{10}$ We have compared single injection transcricoid local anaesthesia with the "spray as you go" technique in patients presenting for day case diagnostic fibreoptic bronchoscopy.

\section{Methods}

Approval for the study was gained from the Wandsworth Health Authority district ethical committee.

We studied patients presenting for routine diagnostic fibreoptic bronchoscopy unless they were having bronchoscopy for haemoptysis with a normal chest radiograph or had stridor or abnormal coagulation or were known to be HIV positive. These exclusion criteria were defined as contraindications for a transcricoid injection. After giving written, informed consent patients were assigned by a randomisation code to receive either transcricoid local anaesthesia (group 1) or "spray as you go" anaesthesia (group 2).

All patients had about $5 \mathrm{ml}$ of $2 \%$ lignocaine gel applied to each nostril. ${ }^{7}$ Group 1 patients received a single transcricoid injection of $5 \mathrm{ml}$ of $2^{\circ}{ }_{\circ}$ lignocaine solution given as a bolus through a 21 gauge needle while they were sitting up. After puncture of the cricothyroid membrane the ability to aspirate air confirmed that the needle position was correct. In group $2,4 \mathrm{ml}$ of $4 \%$ lignocaine was instilled on to the vocal cords in two aliquots under direct vision after insertion of the bronchoscope. A further $2 \mathrm{ml}$ of $2 \%$ lignocaine solution was instilled into each main bronchus. Patients in both groups had a small occlusive plaster affixed over the cricothyroid membrane to ensure that assessment was blind. If local anaesthesia was thought inadequate- for instance, during a biopsyfurther $2 \mathrm{ml}$ boluses of lignocaine were instilled through the bronchoscope. 
Patients were sedated with an intravenous injection of alfentanil $(12 \mu \mathrm{g} / \mathrm{kg}$ for patients under 61 years or $9 \mu \mathrm{g} / \mathrm{kg}$ for patients of 61 or more) and glycopyrrolate $(0.2 \mathrm{mg})$ was given as an anticholinergic agent. ${ }^{6}$ Both were given immediately before insertion of the bronchoscope. If sedation was considered inadequate after five minutes a further dose of alfentanil, equal to half the initial dose, was given.

The time from nasal insertion of the bronchoscope to crossing the vocal cords and from crossing the vocal cords to withdrawal was recorded for all patients. Coughs were counted by an assistant using a mechanical counting device after passage of the bronchoscope across the vocal cords. Where coughs occurred in paroxysms each was counted singly. The differences in the local anaesthetic techniques rendered blinding of the assistant impractical.

After the bronchoscopy the operator recorded the number of endobronchial procedures and an assessment of endotracheal bleeding from the transcricoid puncture site on a standard form.

Just before discharge an assistant, who was unaware of the patient's group, administered a questionnaire and examined the patient's neck (with the sticking plaster in place). The first page of the questionnaire contained questions and a visual analogue scale designed to assess how unpleasant the bronchoscopy was. The scale was labelled "not at all unpleasant" at its origin and "intolerable" at $100 \mathrm{~mm}$. Having been told how to complete the visual analogue scale, all patients were given a second page containing questions and a further visual analogue scale designed to assess the discomfort of the transcricoid injection where applicable. This page was completed in private and handed to the nursing staff as the patient left the endoscopy unit, so that the assessor remained blind.

All quantitative data and an arcsin transformation of the visual analogue scale data ${ }^{11}$ were analysed with an unpaired two tail Student's $t$ test. All qualitative data were analysed with the $\chi^{2}$ test. Values are given as means with standard deviations in parentheses unless specified otherwise.

\section{Results}

Seventy patients were studied (35 in each group). Data were lost for two patients and four patients left the endoscopy unit before being offered the questionnaire. Two patients were withdrawn after the study protocol had been broken. One patient in the transcricoid group had $2 \mathrm{ml}$ of $4 \%$ lignocaine instilled on to the vocal cords inadvertently and one patient in the spray group required additional sedation with benzodiazepine. Thus 32 patients from the spray group and 30 from the transcricoid group were included in the analysis.

The two groups were well matched. There were 18 men in the spray group (mean age 62.5 (SD 15.7) years) and 23 in the transcricoid group (mean age $62 \cdot 2(14 \cdot 2)$ years). The groups
Table 1 Number of patients requiring additional lignocaine

\begin{tabular}{lll}
\hline Site & $\begin{array}{l}\text { Spray group } \\
(n=32)\end{array}$ & $\begin{array}{l}\text { Transcricoid group } \\
(n=30)\end{array}$ \\
\hline Upper lobe & 1 & 7 \\
Main bronchus & 4 & 5 \\
Middle lobe & 1 & 1 \\
Lower lobe & 2 & 2 \\
Total & $8^{\star}$ & $15^{\star}$ \\
\hline
\end{tabular}

${ }^{*} \gamma^{2}=4.15, \mathrm{p}<0.05$

had a similar requirement for sedation, the mean dose of alfentanil being 10.44 (1.64) $\mu \mathrm{g} /$ $\mathrm{kg}$ in the transcricoid group and 10.37 $(1.71) \mu \mathrm{g} / \mathrm{kg}$ in the spray group (none of these differences was significant).

The bronchoscopy took longer in the spray group than in the transcricoid group, the mean time required to traverse the vocal cords being $2.69(2.02)$ minutes in the spray group and 1.35 $(0.98)$ minutes in the transcricoid group $(t=3 \cdot 16, \mathrm{p}<0.01)$. The time taken to complete the bronchoscopy after passage across the vocal cords, however, was similar in the two groups-9.69 (4.8) minutes in the spray group and $9.60(5.8)$ minutes in the transcricoid group $(t=0.06, \mathrm{NS})$.

There were no differences between the numbers or types of endobronchial procedures undertaken in the two groups. Although more patients in the transcricoid group required additional lignocaine (table 1 ), the total doses were much lower than in the spray group (table 2). Despite this, the cough rate was lower in the transcricoid group, the mean rate being 3.56 (3.1) coughs/min compared with $5 \cdot 89(4 \cdot 8)$ in the spray group $(t=2 \cdot 24, \mathrm{p}<0.05)$.

Neither local anaesthetic technique was associated with a more unpleasant bronchoscopy for the patient. Seven of the 30 patients in the spray and three of the 32 in the transcricoid group recorded that they did not find the procedure unpleasant and 15 patients from each group found the procedure only slightly unpleasant (figure). After arcsin transformation of the visual analogue scale data the scores were similar, being 23.7 (25) $\mathrm{mm}$ in the spray group and $24.8(23) \mathrm{mm}$ in the transcricoid group ( $t=0 \cdot 186, \mathrm{NS})$.

Of the 30 patients who received a transcricoid injection, 17 found it "not unpleasant"

Table 2 Location and mean (SD) doses of lignocaine ( $m g$ ) used in the study

\begin{tabular}{lcl}
\hline & $\begin{array}{l}\text { Spray group } \\
(n=32)\end{array}$ & $\begin{array}{l}\text { Transcricoid group } \\
(n=30)\end{array}$ \\
\hline $\begin{array}{l}\text { Nasal gel } \\
\text { Vocal cords }\end{array}$ & 200 & 200 \\
$\begin{array}{l}\text { Transcricoid } \\
\text { Bronchial tree }\end{array}$ & 160 & 100 \\
$\begin{array}{l}\text { Additional lignocaine } \\
\text { Total dose }\end{array}$ & $11(20.9)^{\star}$ & $22(25 \cdot 9)^{\star}$ \\
Total dose below nose & $451(20.9) \dagger$ & $322(25.9) \dagger$ \\
\hline
\end{tabular}

${ }^{\star} t=1 \cdot 80, \mathrm{NS}$.

$+t=21.67, \mathrm{p}<0.001$. 

experienced by patients.
No of patients

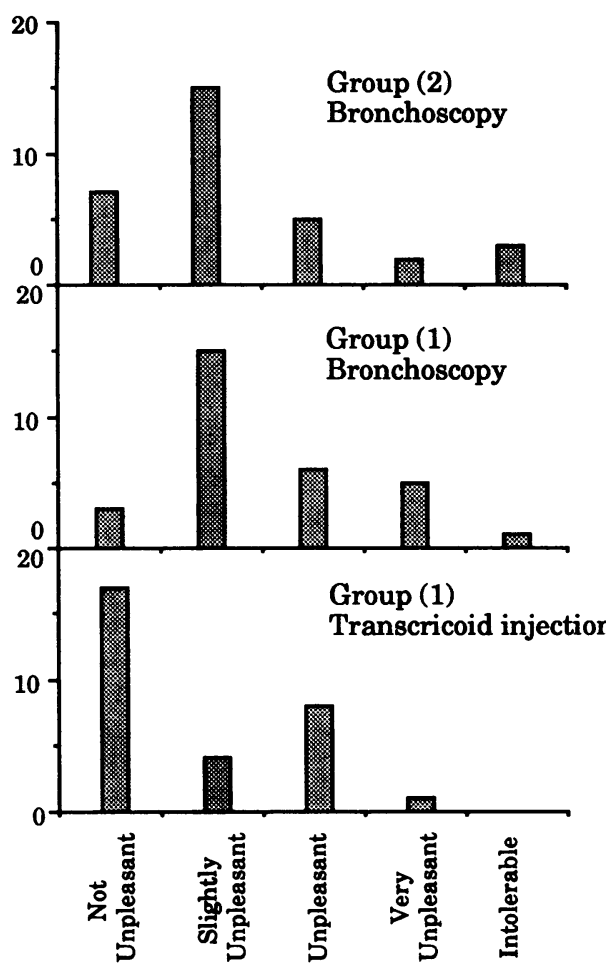

and only one patient found it "very unpleasant." With the same visual analogue scale and arcsin transformation, the mean score was $13.6(15 \cdot 1) \mathrm{mm}$. The transcricoid injection was not associated with any complications. There was blood in the trachea in 23 of the 30 patients but it did not interfere with the bronchoscopy in any patient. The amount of bleeding was quantified by the operator as a trickle in 16 , reaching the carina in five and reaching a main bronchus in two. There were no cases of haematoma or local swelling and no cases of subcutaneous emphysema when the neck was examined blind after the procedure.

\section{Discussion}

The study set out to compare two commonly used techniques of applying topical anaesthesia to the respiratory mucosa for diagnostic fibreoptic bronchoscopy. The two groups were well matched for age, sex, sedation, and endobronchial procedures and therefore allowed a fair comparison of the two techniques being studied.

The transcricoid method was more effective than the "spray as you go" method. Despite the lower dose of lignocaine the duration of the bronchoscopy was shorter, the cough rate was lower, and the procedure was at least as acceptable to the patients. Twice as many patients required additional aliquots of lignocaine in the transcricoid group-usually just before endobronchial procedures, particularly in the upper lobes. Where additional lignocaine

was required the mean additional dose was similar in the two groups. A similar reduction in cough with the transcricoid method has been reported (in abstract form) by Kinnear et al.

The major problem in comparisons of different methods of applying topical anaesthesia to the tracheobronchial mucosa is that we do not know where the lignocaine is deposited. The application of lignocaine by the transcricoid route is associated with an initial cough, during which some lignocaine may be lost from the respiratory tract. Similarly, we do not know what proportion of lignocaine instilled on to the vocal cords through the bronchoscope is lost to the gastrointestinal tract. We may speculate whether equivalent starting dosages of lignocaine in the two groups would have accentuated or minimised the differences between the groups.

Coughing during fibreoptic bronchoscopy not only is unpleasant for the patient, particularly as the foreign object in the trachea cannot be coughed out, but also renders operating conditions difficult, with recurrent spasmodic movement of structures that are being viewed or operated on. Even though the transcricoid injection often produces a cough, it is short lived and not associated with the unpleasant sensation of not being able to eject a foreign object. As the primary reason for topical anaesthesia of the respiratory mucosa is to reduce cough, the reduced rate of cough (with lower dosage of local anaesthetic) produced by the transcricoid technique is a clear advantage. We did not attempt to measure the strength of cough as this would have been highly subjective in an unblinded observer.

There was a minor but significant reduction in the total time of the bronchscopy. This is adväntageous to our practice, because nasal anaesthesia and transcricoid anaesthesia are performed in an anteroom while the bronchoscopy room is being prepared between patients. We have noted a quicker turn around of patients with the transcricoid technique as anteroom procedures take longer and the bronchoscopy follows without delay. With the "spray as you go" technique the shorter time needed in the anteroom is often followed by a delay until the bronchoscopy room is ready and the bronchoscopy takes longer.

Four of the authors were not familiar with the transcricoid technique before the study, but learning was quick and a preference for the technique has been expressed. The bronchoscopy was not particularly unpleasant for the patients in either group and coughing was the most common reason for any unpleasantness in both groups. The transcricoid injection was performed before sedation of the patient because cooperation was required for correct positioning. Despite this the technique was not found to be particularly unpleasant for the patient.

There were no complications associated with transcricoid injection and the minor bleeding associated with the technique did not interfere with the bronchoscopy.

In conclusion, the transcricoid technique can be recommended as a safe method of inducing 
effective local anaesthesia that is well tolerated by the patient.

We would like to thank the staff of the endoscopy unit at St George's Hospital for their support and cooperation and Dr F J C Millard for his help in setting up the study.

1 Kortilla $\mathrm{K}$, Tarkkanen J, Tarkkanen L. Comparison of Laryngotracheal and Ultrasonic Nebulizer Administration of Lidocaine in Local Anaesthesia for Bronchoscopy. Acta Anaesth Scand 1981;25:161-5.

2 Credle WF, Smiddy JF, Elliot RC. Complications of fibreoptic bronchoscopy. Am Rev Respir Dis 1974;109: 67-72.

3 Perry LB. Topical anesthesia for bronchoscopy. Chest 1978;73(suppl):691-3.

4 Patterson JR, Blaschke TF, Kellogg Hunt K, Meffin PJ.
Lidocaine blood concentrations during fiberoptic bronchoscopy. Am Rev Respir Dis 1975;112:53-7.

5 Jones DA, McBurney A, Stanley PJ, Tovey C, Ward JW. Plasma concentrations of lignocaine and its metabolites during fibreopti

6 Webb AR, Doherty JF, Chester MR, et al. Sedation for fibreoptic bronchoscopy: comparison of alfentanil with papaveretum and diazepam. Respir Med 1989;83:213-7.

7 Webb AR, Woodhead MA, Dalton HR, Grigg JA, Millard FJC. Topical nasal anaesthesia for fibreoptic bronchoscopy: patients' preference for lignocaine gel. Thorax 1989;44:674-5

8 Macdonald JB. Fibreoptic bronchoscopy today: a review of 255 cases. Br Med J 1975; iii:753-5.

9 Macfarlane JT, Ward MJ. Transtracheal injection of saline in the investigation of pneumonia. $\mathrm{Br} M e d J$ 1984; 288:974-5.

10 Fry WA. Techniques of topical anesthesia for bronchoscopy. Chest 1978;73(suppl):694-6.

11 Aitken RC. A growing edge of feelings. Proc $R$ Soc Med 1969;62:989-93. 